\title{
Incidence Patterns and Outcomes for Hodgkin Lymphoma Patients in the United States
}

\author{
Pareen Shenoy, Alison Maggioncalda, Neha Malik, and Christopher R. Flowers \\ Winship Cancer Institute, Emory University, 1365 Clifton Road, N.E. Building B, Suite 4302, Atlanta, GA 30322, USA \\ Correspondence should be addressed to Christopher R. Flowers, crflowe@emory.edu
}

Received 2 July 2010; Accepted 19 October 2010

Academic Editor: Emili Montserrat

Copyright () 2011 Pareen Shenoy et al. This is an open access article distributed under the Creative Commons Attribution License, which permits unrestricted use, distribution, and reproduction in any medium, provided the original work is properly cited.

Hodgkin lymphoma (HL) demonstrates heterogenous histologic findings, clinical presentation, and outcomes. Using the United States Surveillance, Epidemiology, and End Results (SEER) data we examined relationships between patient characteristics, clinical features at diagnosis, and survival in HL patients. From 2000 to 2007, 16,710 cases were recorded in 17 SEER registries. Blacks and Asians had low incidence (black/white incidence rate ratio (IRR) $0.86, P<.01$; Asian/white IRR $0.43, P<.01$ ). The bimodal pattern of incidence was less prominent for black males. Asians and Blacks presented at a mean age of 38 years compared to 42 years for Whites $(P<.001)$. Race was a predictor for survival with HR of 1.19 (95\% CI 1.11-1.28) for Blacks. Age was the most important predictor of survival (HR for patients $\geq 45$ years $5.08,95 \%$ CI $4.86-5.31$ ). These current patterns for presentation and outcomes of HL help to delineate key populations in order to explore risk factors for HL and strategies to improve treatment outcomes.

\section{Introduction}

Hodgkin lymphoma (HL) is a cancer of the lymphatic system commonly characterized by the presence of large malignant lymphoid cells, Reed-Sternberg cells, although malignant cells typically account for less than $1 \%$ of cells in the affected tissue [1]. The variable quantity and quality of the malignant cells and the host response make the histologic findings in HL heterogenous [2]. In the United States (US), an estimated 8,490 new cases were diagnosed with HL while HL was accountable for 1,320 deaths in 2010 [3]. In the 1960s, the 5 -year survival rate for HL was less than $10 \%$ [4]. With breakthroughs in combination chemotherapy regimens, the reported 5-year survival for patients with HL during the years $2000-2004$ was $85.2 \%$ [5]. However, relatively little is known regarding the clinical and demographic factors that influence the patterns of presentation and outcomes for HL in a population-based setting in the US.

Recent epidemiological studies have found independent associations between age, gender, race, geographic location, and incidence of HL [2]. For example, Asians have been shown to have a dramatically lower HL incidence than other races, but even among US Asians there are significant incidence rate differences between US-born Asians versus native Asians [6]. The incidence variation by age, geographic location, social class, and time suggests an etiologic role for infectious agents, such as Epstein-Barr virus (EBV), while aggregation in families and persons with specific human leukocyte antigen (HLA) types indicates genetic susceptibility $[2,7]$. These and other factors influencing HL incidence and outcomes are reviewed elsewhere in this journal [7]. To examine the relationships between demographic patient characteristics, clinical features at diagnosis, and survival outcomes in a national cohort of patients with HL, we performed a retrospective analysis of HL cases diagnosed from 1973 to 2007 reported to population-based cancer registries.

\section{Methods}

2.1. Data Sources. We obtained population-based cancer incidence data from the United States Surveillance, Epidemiology, and End Results (SEER) Program. This database has compiled incidence data since 1973 from nine populationbased registries (Connecticut, Hawaii, Iowa, New Mexico, 
Utah, Detroit, San Francisco/Oakland, Seattle/Puget Sound, and Atlanta), accounting for $10 \%$ of the United States population [8]. The SEER 9 registries population is comparable to the general population except containing a higher proportion of urban areas and foreign-born persons [9]. In 1992, SEER expanded to include two additional metropolitan areas (Los Angeles County and San Jose/Monterey, California), Rural Georgia, and the Alaska Native Tumor Registry, together accounting for approximately $14 \%$ of the United States population $[10,11]$. Later in 2000, SEER included Greater California, Kentucky, Louisiana, and New Jersey registries to account for $26 \%$ of the US population [12]. For the analyses of incidence rates, we used the SEER17 data compiled of cases diagnosed from January 1, 2000 to December 31, 2007 [12]. Given its longer duration of followup, for the survival analysis, we used the SEER9 data compiled of cases diagnosed from January 1, 1973 to December 31, 2007 [7].

2.2. Study Cohort. The classification of lymphoid neoplasms has undergone several updates since the 1940s. Lymphomas diagnosed from 1973 through 1977 were classified according to the Manual of Tumor Nomenclature and Coding [13]. Then in 1978, the International Classification of Diseases for Oncology (ICD-O) [14] was adopted to code all cancers registered by the SEER Program. In 1992, the SEER Program adopted the ICD-O, 2nd Edition (ICD-O-2) to code lymphomas [15]. In 2001, the World Health Organization (WHO) classification was introduced building on the Revised European-American Lymphoma classification and the French-American-British classification $[16,17]$ and updated in 2008 [18]. Recently the United States cancer registries adopted the ICD-O-3 with the ability to convert cases coded in ICD-O-2 to ICD-O-3 [19] making it feasible to assess incidence patterns and trends for lymphoid neoplasms according to the internationally recognized WHO classification [20].

We identified and categorized HL cases using the third edition of the International Classification of Diseases for Oncology (ICD-O-3) [21] into classical HL (CHL) and nodular lymphocyte predominant HL (NLP, ICD-O-3 9659). CHL cases were further divided into the following subtypes: lymphocyte-rich (LR, 9651), mixed cellularity (MC, 9652), lymphocyte-depleted (LD, 9653-9655), nodular sclerosis (NS, 9663-9667), and not-otherwise-specified (NOS, 96509651) (Table 1). This corresponds with the InterLymph clustering of the WHO classification of lymphoid malignancies into categories designed for lymphoma epidemiological research [22]. All data refer to the incidence of neoplasms with malignant behavior. The selection of HL cases for analysis of incidence and survival is depicted in Figures 1(a) and $1(\mathrm{~b})$, respectively. Cases with unknown or unspecified race were excluded from the analysis. Cases with known race were grouped into white, black, American Indians or Alaska Natives (AI/ANs), and Asians or Pacific Islanders (A/PIs). The SEER program records race as assigned by the North American Association of Central Cancer Registries [23]. Race and ethnicity were defined by specific physical, hereditary, and cultural traditions or origins, not necessarily by birthplace, place of residence, or citizenship. Examination of Hispanic ethnicity was beyond the scope of this study because ethnicity was not recorded reliably in the SEER registries during this time period [24].

SEER data were used to calculate survival time using the date of diagnosis and one of the following: date of death, date last known to be alive, or date of the study cutoff (December $31,2007)$. For survival analyses, patients whose disease status was based on a death certificate or autopsy only, patients with second or later primaries, and patients who were not actively followed were excluded, resulting in a cohort of $(23,292)$ cases. To evaluate the impact of modern therapies on survival, we divided patients into eras by year of diagnosis: 1973-1979, 1980-1989, 1990-1999, and 2000-2007.

2.3. Calculation and Presentation of Rates. Incidence rates were expressed as new cases per 100,000 person-years and age-adjusted to the 2000 U.S. standard population [25]. Incidence rates were compared by sex and race using incidence rate ratios (IRRs) and $95 \%$ confidence intervals (95\% CIs). Differences in baseline characteristics at diagnosis across racial groups were analyzed using two-sided $t$-tests and chi-square tests.

Two-year and five-year relative survival rates (RSRs) were calculated by actuarial methods, where relative survival is defined as the ratio of the proportion of observed survivors in a cohort of cancer patients to the proportion of expected survivors in a comparable set of cancer-free individuals, thus representing survival in the absence of other causes of death. A $Z$-test was used to test the equivalence of RSRs [26]. Survival curves were constructed using the Kaplan-Meier method and compared with the log-rank test. Univariate and multiple variable Cox proportional hazards models were used to examine the covariates age at diagnosis, gender, race, stage of disease, presence of extranodal disease, and presence of B-symptoms as predictors of mortality.

A level of significance $(\alpha)$ of .05 was considered statistically significant. All statistics were computed using the National Cancer Institute SEER*Stat software, version 6.5.2. (http://www.seer.cancer.gov/seerstat/), SAS software, version 9.0 (SAS Institute Inc. Copyright (C)2002), and STATA 9.2 (StataCorp LP. Copyright (C)1855-2006).

\section{Results}

3.1. Incidence of $H L$. During the period from 2000 to 2007, 16,710 cases of HL were diagnosed and reported to the 17 SEER registries. Of these, 161 cases with unknown/unspecified race were excluded resulting in a study cohort of 16,549 cases: 14,076 whites, 1,693 blacks, $58 \mathrm{AI} / \mathrm{AN}$, and $722 \mathrm{~A} / \mathrm{PI}$. The study populations for the incidence and survival analyses are shown in Figure 1. The age-adjusted incidence rate for HL was 2.74 (95\% CI 2.69-2.78), NLP was 0.11 (95\% CI $0.1-0.12)$, and for CHL was 2.63 (95\% CI 2.59-2.67). The age-adjusted incidence rates for the CHL subtypes LR, MC, LD, NS, and CHLNOS were 0.08 (95\% 


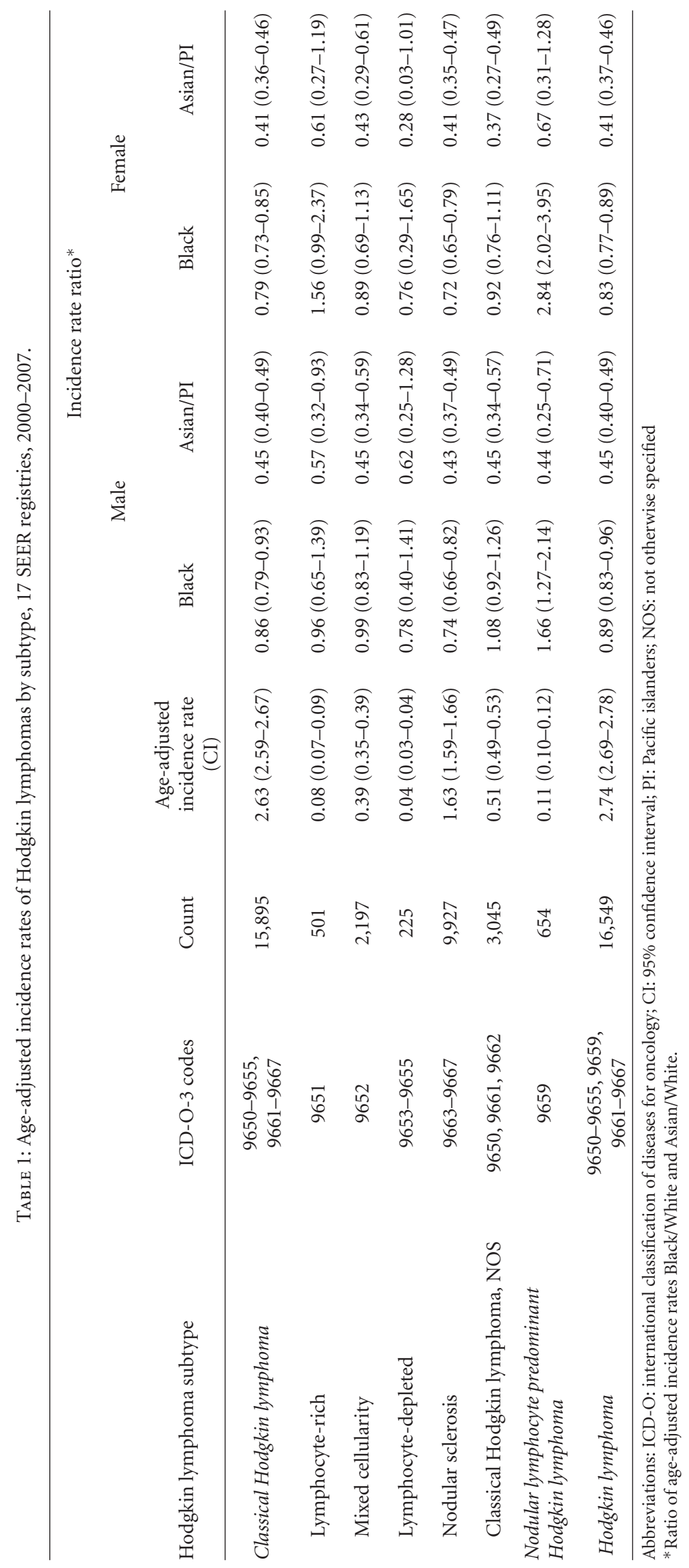




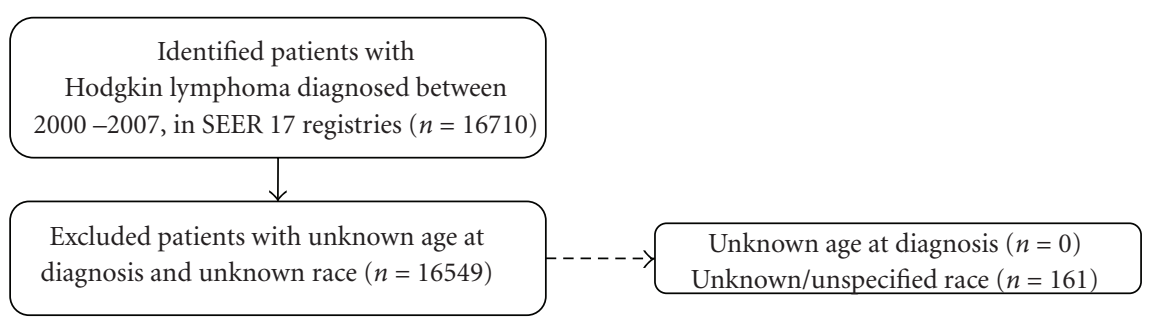

(a)

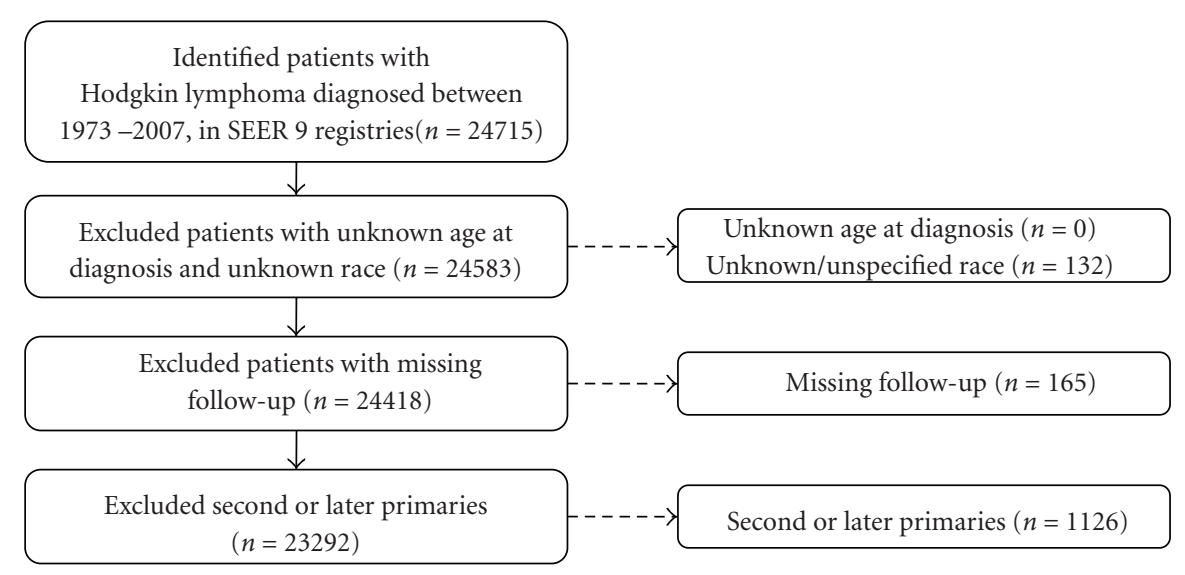

(b)

Figure 1: Selection of study cohort. These figures provide an overview of the study cohort with reasons for inclusion/exclusion through the selection process. (a) Selection of the cases included in the incidence analyses. (b) Selection of the cases included in the survival analyses.

CI 0.07-0.09), 0.37 (95\% CI 0.35-0.39), 0.04 (95\% CI $0.03-$ 0.04), 1.63 (95\% CI 1.59-1.66), and 0.51 (95\% CI 0.49-0.53), respectively (Table 1$)$.

3.2. Incidence Rates by Sex and Race. Males had significantly higher incidence rates than females for all HL subtypes. The male-to-female (M/F) IRR was 1.27 (95\% CI 1.23-1.31) for all HL combined and greatest for NLP HL $(2.45,95 \%$ CI 2.12.9). Male predominance among HL was most pronounced among A/PI (M/F IRR 1.35, 95\% CI 1.2-1.6). Although the overall incidence rates for HL were significantly lower for black patients than white patients (black-to-white IRR 0.86, $P<.01)$, black patients had higher incidence rates compared to white patients for NLP HL (black-to-white IRR 1.9, $P<$ .01; Table 1). The difference in incidence between whites and Asians was even more prominent (Asian-to-white IRR 0.43, $P<.01)$.

3.3. Age-Specific Incidence Rates. The age distribution of $\mathrm{CHL}$ across gender and race differed. White patients demonstrated a bimodal distribution with prominent peaks at age 21-30 and >70 years, although the second peak for white females was less prominent (Figure 2(a)). This bimodal age distribution pattern was seen in all groups except for black males with CHL. For NLP HL, however, the only obvious bimodal incidence pattern was seen for black males, with peak incidence rates at 31-40 years and 51-60 years.
3.4. Baseline Characteristics at Diagnosis. The baseline characteristics at diagnosis by race are compared in Table 2 . The mean age at diagnosis for black patients and Asian/PI was younger than that for white patients (both $P<.001$; Table 2 ). Blacks had a higher percentage of cases with an extranodal disease $(27 \%$ versus $23 \%, P<.001)$, but no significant difference in the percentage of patients with $B$ symptoms (36\% versus $35 \%, P=.058$ ) compared to white patients. Compared to white patients, black patients had a higher percentage of cases diagnosed with stage III/IV disease $(43 \%$ versus $35 \%, P<.001)$. Asian/PI patients also had a slightly higher percentage of cases with stage III/IV HL (38\% versus $35 \%, P<.001)$. Information on stage, extranodal disease, and B-symptoms was missing in $5.7 \%, 5.7 \%$, and $24 \%$ of cases, respectively.

3.5. Survival Analysis. The 2-year and 5-year RSRs for the entire population of patients HL were $87 \%$ and $80 \%$. The 2-year RSRs for CHL subtypes NS; LR; MC; LD; CHL, NOS; NLP HL were 92\%, 93\%, 81\%, 49\%, 75\%, and 97\%, respectively. The 5 -year RSRs for CHL subtypes NS; LR; MC; LD; CHL, NOS; NLP HL were 86\%, 87\%, 73\%, 43\%, 68\%, and $94 \%$, respectively. Female patients had better RSRs than males (2-year RSR: $88 \%$ versus $86 \%$, 5 -year RSR: $83 \%$ versus $78 \%$, both $P<.001)$. White, black, and A/PI patients had similar survival rates (2-year RSR: $87 \%, 85 \%$, and $84 \%$, resp., 5-year RSR: $81 \%, 77 \%$, and 77\%, resp., $P<.01)$. However, race was a significant predictor of survival in Cox regression 


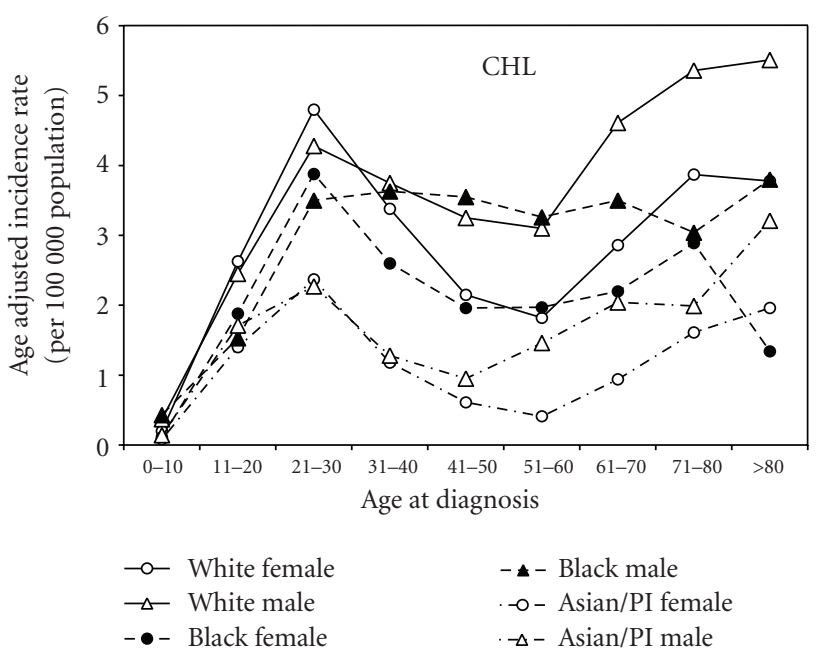

(a)

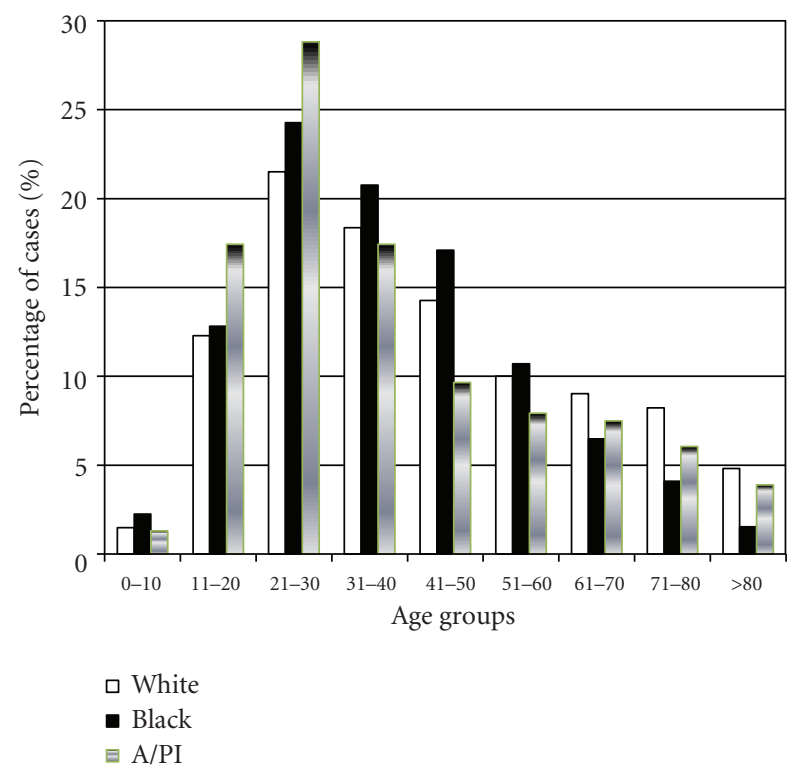

(c)

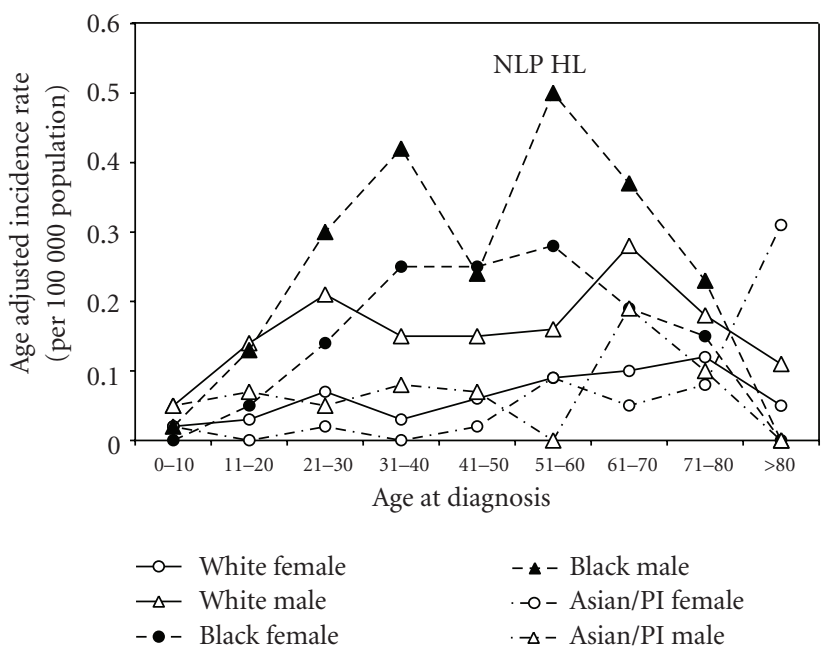

(b)

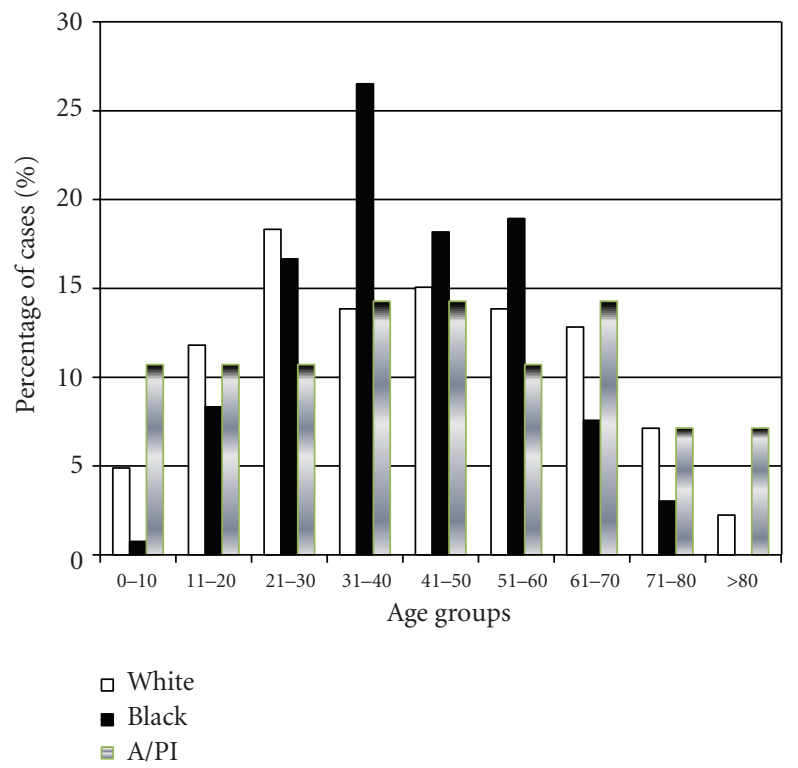

(d)

Figure 2: Age distribution of HL by race. (a) Plots are the race- and gender-specific age-adjusted incidence rates of CHL by age at diagnosis. (b) Plots are the race- and gender-specific age-adjusted incidence rates of NLP HL by age at diagnosis. (c) Plots are the age distribution of CHL patients. Horizontal axis represents the grouping of age at diagnosis. Vertical axis represents the proportion of patients in each age group for that particular race (White, Black, and Asian/Pacific Islander). (d) Plots are the age distribution of NLP HL patients. Horizontal axis represents the grouping of age at diagnosis. Vertical axis represents the proportion of patients in each age group for that particular race (White, Black, and Asian/Pacific Islander).

models (Table 3). In multiple variable Cox regression models, the most significant predictors of survival were age $\geq 45$ (HR 4.82, 95\% CI 4.40-5.29), B symptoms (HR 1.82, 95\% CI 1.66-2.00), black race (HR 1.54, 95\% CI 1.35-1.76), stage IV disease (HR 1.29, 95\% CI 1.05-1.59), and male gender (HR 1.17, 95\% CI 1.07-1.28; Table 3). Multiple variable Cox regression models demonstrated that when compared to NS HL, MC HL, LD HL, and CHL, NOS had worse survival with HR of 1.31 (95\% CI 1.17-1.46), 2.02 (95\% CI 1.58-2.57), and 1.65 (95\% CI 1.46-1.86), respectively (Table 3 ).
There was a statistically significant improvement in survival across consecutive diagnostic eras (log-rank test $P<$ .001 , Figure $3(\mathrm{c})$ ) with similar trends observed for each racial group. The improvement in survival across the diagnostic eras was also seen uniformly in all subtypes. The 5-year RSR for NLP HL was $71.5 \%$ for patients diagnosed in the years 1973-79; 91.8\% (1980-89); 91.5\% (1990-99); 98.9\% (2000-07). Those for NS HL were $80.1 \%$ (1973-79); 84.2\% (1980-89); 88\% (1990-99); 89.9\% (2000-07). Kaplan-Meier survival curves also show a significant difference in survival 
TABLE 2: Racial differences in the presentation of Hodgkin lymphoma.

\begin{tabular}{|c|c|c|c|c|c|c|c|c|}
\hline \multirow{2}{*}{ Characteristic } & \multicolumn{2}{|c|}{ White } & \multicolumn{2}{|c|}{ Black } & \multicolumn{2}{|c|}{$\mathrm{A} / \mathrm{PI}$} & \multicolumn{2}{|c|}{$P$-value } \\
\hline & Count & $\%$ & Count & $\%$ & Count & $\%$ & $W$ versus $B$ & $\mathrm{~W}$ versus $\mathrm{A} / \mathrm{PI}$ \\
\hline$n$ & 14,076 & & 1,693 & & 722 & & & \\
\hline \multicolumn{9}{|l|}{ Age, years } \\
\hline Mean & 42 & & 38 & & 38 & & $<.001$ & $<.001$ \\
\hline Interquartile Range & $26-57$ & & $25-49$ & & $23-51$ & & & \\
\hline$<45$ & 8,472 & 60.19 & 1,148 & 67.81 & 493 & 68.28 & $<.001$ & $<.001$ \\
\hline$\geq 45$ & 5,604 & 39.81 & 545 & 32.19 & 229 & 31.72 & & \\
\hline Male sex & 7,711 & 54.78 & 913 & 53.93 & 399 & 55.26 & .51 & .80 \\
\hline \multicolumn{9}{|l|}{ Stage } \\
\hline I & 2,717 & 19.30 & 360 & 21.26 & 95 & 13.16 & $<.001$ & $<.001$ \\
\hline II & 5,555 & 39.46 & 527 & 31.13 & 327 & 45.29 & & \\
\hline III & 2,639 & 18.74 & 365 & 21.56 & 133 & 18.42 & & \\
\hline IV & 2,349 & 16.69 & 356 & 21.03 & 128 & 17.73 & & \\
\hline Unknown & 816 & 5.80 & 85 & 5.02 & 39 & 5.40 & & \\
\hline \multicolumn{9}{|l|}{ Extranodal disease } \\
\hline Yes & 3,170 & 22.52 & 455 & 26.88 & 179 & 24.79 & $<.001$ & .17 \\
\hline No & 10,090 & 71.68 & 1,153 & 68.10 & 504 & 69.81 & & \\
\hline Unknown & 816 & 5.80 & 85 & 5.02 & 39 & 5.40 & & \\
\hline \multicolumn{9}{|l|}{ B symptoms } \\
\hline Yes & 4,988 & 35.44 & 608 & 35.91 & 305 & 42.24 & .06 & .60 \\
\hline No & 5,783 & 41.08 & 629 & 37.15 & 275 & 38.09 & & \\
\hline Unknown & 3,305 & 23.48 & 456 & 26.93 & 142 & 19.67 & & \\
\hline
\end{tabular}

Note: for each variable, cases with unknown information were excluded in calculating the $P$-value.

Abbreviations: W: whites; B: blacks; O: others.

by age at diagnosis, with patients who were $\geq 45$ years having a worse outcome (log-rank test $P<.001)$. Across histologic subtypes, survival for patients with NLP HL was better than that for patients with CHL (log-rank test $P<.001$; Figure $3(d))$. Although stage of disease did affect survival outcomes, age $\geq 45$ years had a greater impact on survival (Figure 3(b)).

\section{Discussion}

Our study examines incidence, presentation, and survival of HL with respect to previously assessed risk factors: age, gender, race, stage, and histologic subtype. Although these risk factors have been examined in prior studies, the majority of these studies focused on one factor independent of the others. Using the most recent national SEER data, we analyzed the relationships between patient demographics, presentation of disease, and overall survival in an attempt to identify which risk factors had the most influence on incidence and survival for HL.

Lack of central pathology review is a limitation of this and all other SEER studies. In our study cohort, patients included were diagnosed with HL based on local healthcare system standards. Another possible limitation of this analysis is the use of cases classified according to ICD-O-2, prior to the introduction of the revised WHO classification in 2001.
Clarke et al. studied the reliability of computer-converted ICD-O-2 codes to ICD-O-3 codes in SEER and found that the agreement for classical HL was 95\% while that for nodular HL was $44 \%[19,27]$.

In the current analyses, we categorize racial groups into white, black, AI/AN, and A/PI. It should be noted, however, that clumping all Asians together might not be meaningful for detailed epidemiologic studies since it lumps together genetically distinct and heterogenous populations of patients who would likely have different genetic, dietary, and possibly environmental risks for lymphoma. Although the SEER data provide detailed information on race at the case level, it classifies race into white, black, $\mathrm{AI} / \mathrm{AN}$, and A/PI at the population level; thereby precluding further analyses of racial subgroups with this dataset. Another limitation of the SEER data is the absence of information on the HIV status of the patients. This precludes that analysis of incidence of HIVpositive patients across racial groups.

4.1. Incidence. As expected, HL incidence was higher in males than females and higher in whites than other races. These data also address the debate over the existence of a bimodal incidence pattern for HL with current populationbased data. As Figure 2 displays, there are clear CHL incidence peaks for young adults and individuals $\geq 70$ years of age for both genders and all racial groups except for black 
TABLE 3: Cox regression models of predictors mortality among Hodgkin lymphoma patients.

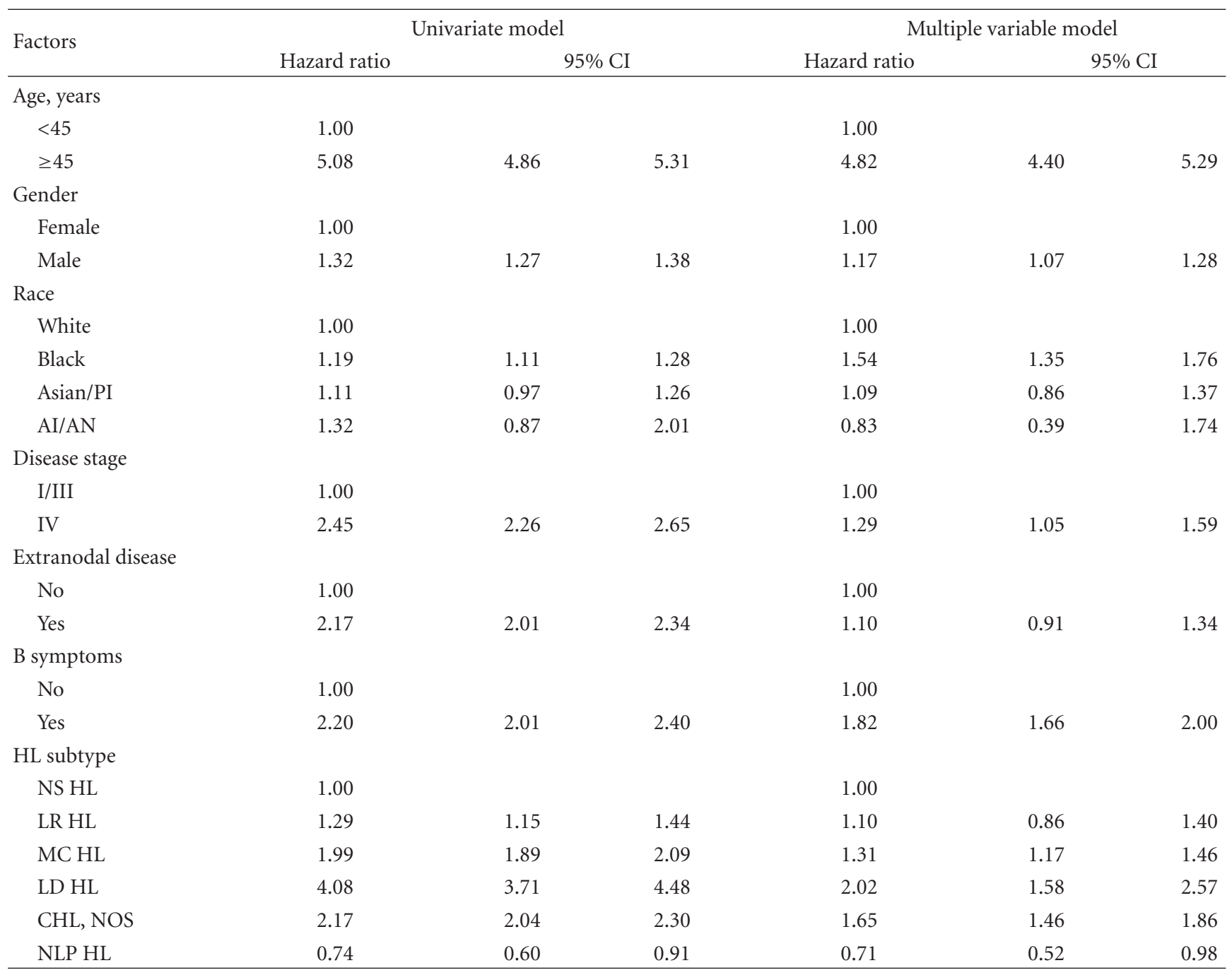

males, where the peaks are not nearly as prominent. While a bimodal incidence pattern of HL is largely debated, these findings are consistent with several other studies [28-30].

In terms of race, both blacks and Asian/Pacific Islanders had lower incidence rates than whites for CHL. Asians had significantly lower incidence rates for virtually all HL subtypes. Internationally, Asians have lower HL incidence than other races. According to Glaser and Hsu, consistently low incidence rates of HL in the Asian population as a whole suggest genetic resistance, possibly related to HLA type, and differences between US-born and native Asian groups suggest environmental influences [6]. HLA type has been shown to affect EBV-related HL incidence, and EBV-positive HL is most common in the Asian population $[31,32]$. Though its impact seems dependent on age, early microbiome exposure has been linked to having a protective effect against HL $[33,34]$. Racial differences in early exposure to viral pathogens and microbiomes may influence these differences in the incidence of HL and the patterns of HL presentation discussed below. For most HL subtypes, black race was associated with lower HL incidence rates. However, for NLP HL, blacks had a significantly higher incidence rate than whites. This is somewhat surprising given that NLP HL has a clinical behavior similar to indolent non-Hodgkin lymphomas (NHLs), and black Americans also have lower incidence of NHL [35]. Further studies investigating the etiology of racial differences in incidence patterns for HL using admixture mapping will help to elucidate genetic susceptibility and gene-environment interaction associated with the incidence of HL and HL subtypes.

4.2. Presentation. We found significant differences in the clinical features at presentation across the racial groups studied. These differences may contribute to racial differences in survival since age of onset, stage, and B-symptoms at diagnosis are known prognostic factors for HL. Not only did blacks and Asian/PI present at a lower age than whites ( 38 versus 42 years), but both groups also had a higher percentage of patients with stage III/IV disease. Additionally, blacks had a higher percentage of patients presenting with an extranodal disease. One possible explanation for these 


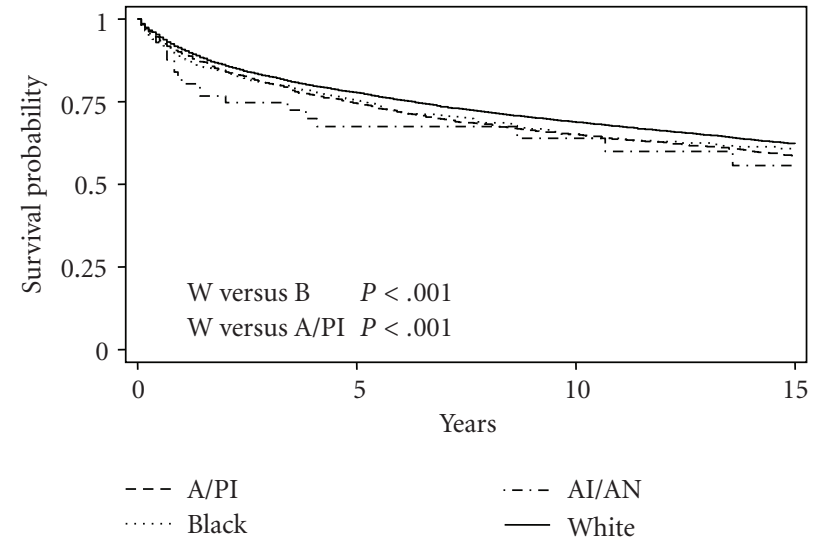

(a)

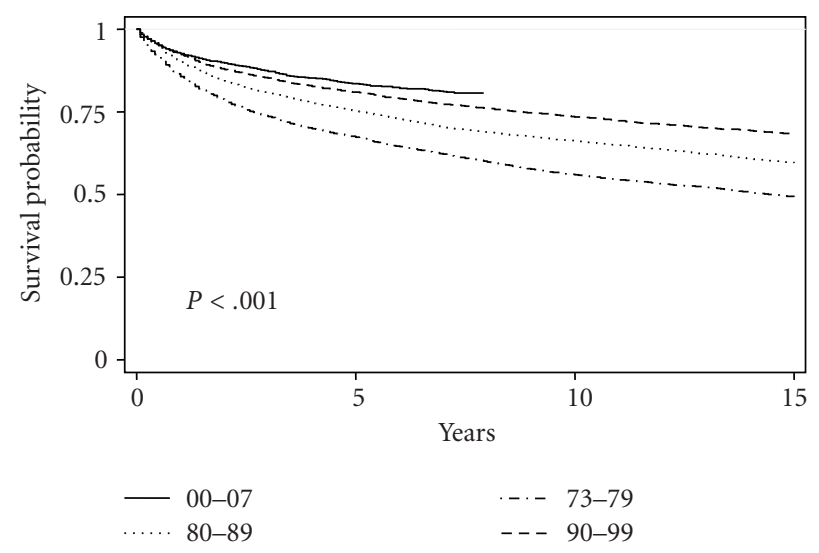

(c)

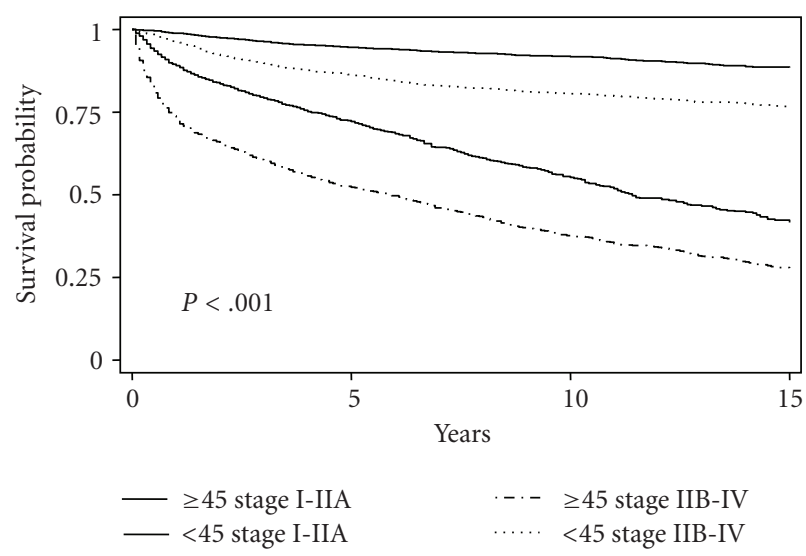

(b)

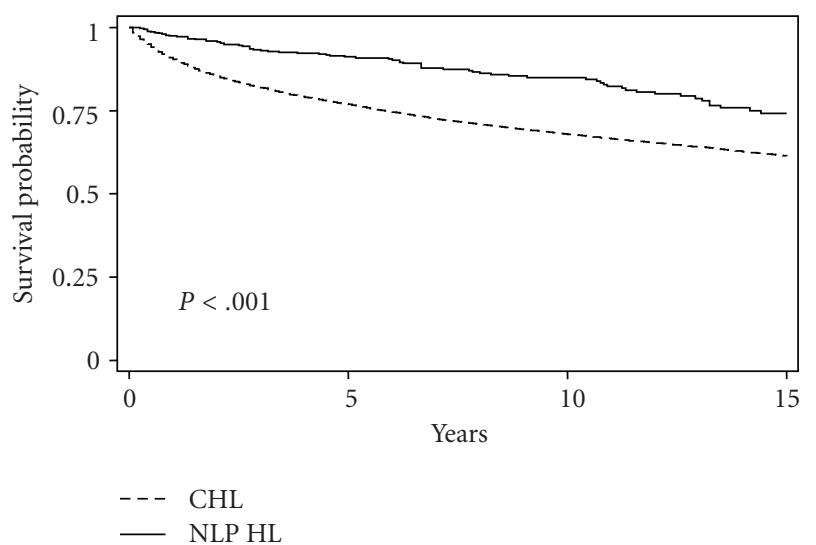

(d)

Figure 3: Kaplan-Meier Survival Curves of HL Patients. (a) Survival curves by race. (b) Survival curves by age at diagnosis ( $<45$ versus $\geq 45$ years) and stage (Stage I-IIA versus Stage IIB-IV). (c) Survival curves by era of diagnosis. (d) Survival curves for CHL and NLP HL.

differences in presentation could be racial differences in the prevalence of human immunodeficiency virus (HIV) among individuals with HL. HIV-positive HL patients present at a more advanced stage with associated extranodal involvement and B symptoms [36]. Biggar et al. found that individuals with HIV/AIDS were also more likely than the general population to be diagnosed with HL. AIDS cases with moderate immunosuppression (225-249 CD4 cells/ $\mu \mathrm{L}$ at onset) were estimated to have a 15-fold increased risk of HL [37]. In Glaser's HIV related HL study, $21.3 \%$ of blacks in the study were HIV positive, whereas only $13 \%$ of whites and $2.3 \%$ of Asians were HIV-positive [36]. On a national scale, blacks are significantly overrepresented in HIV/AIDS incidence [38]. Patients of nonwhite race were diagnosed earlier and with a higher level of disease progression. Recent epidemiological studies in NHL also found significant differences between blacks and whites in the age of presentation and racial differences in overall survival $[35,39-41]$.

4.3. Survival. Age, gender, presenting symptoms, stage, and race were all predictors of survival according to our multiple variable Cox regression. The international prognostic score for advance stage HL identified seven factors with similar independent prognostic effects: serum albumin level $<4 \mathrm{~g}$ per deciliter, hemoglobin level $<10.5 \mathrm{~g}$ per deciliter, male sex, an age of $\geq 45$ years, stage IV disease, white-cell count $>15,000$ per cubic millimeter, and lymphocyte count $<600$ per cubic millimeter, $<8 \%$ of the white-cell count, or both [42]. In this population-based analysis, age of 45 or above, stage IV disease, and male gender remained significant predictors of HL survival for patients with advanced stage disease (data not shown) and all patients. The remaining components of the prognostic score are laboratory based and therefore not able to be analyzed with SEER.

Our data showed that an age of 45 or older had an overwhelming association with mortality (HR 5.25). The next highest predictor was the presence of B symptoms (HR 1.90), followed by black race (HR 1.54). Only after these factors did stage IV disease (HR 1.39) and male gender (1.19) come into play. Age was significantly more predictive of survival than stage (Figure 3(b)). Interestingly enough, both race and B-symptoms at presentation also were significant predictors of survival in multiple variable regression models. 
Determining whether the impact of race and B-symptoms are confounded by differences in the prevalence of HIV or EBV in these groups needs to be addressed with additional studies, since both viruses can influence the incidence and survival of HL $[34,36]$.

As mentioned previously, black individuals also have a higher incidence than whites of NLP HL. Using NS as a reference, MC, LD, and CHL NOS subtype diagnoses were predictive of worse 5-year survival (Table 3 ). Cox regression models showed that NLP was the only subtype that predicted for better survival than NS (Table 3 ), and the overall survival curve (Figure $3(\mathrm{~d})$ ) suggests that while NLP HL may have worse initial outcomes, long-term survival is better for NLP HL patients than for CHL patients. It should however be noted while interpreting these survival analyses that there might be potential misdiagnosis of NLP HL as T-cellrich B-cell lymphoma or follicular lymphoma. Additional HL subtype-specific studies are needed to investigate factors that influence outcomes particularly with the future development of individualized treatment strategies for HL subtypes.

Finally, the survival curves in Figure 3(c) demonstrate that the survival probability continues to improve for patients with HL across eras of diagnosis. This is particularly interesting since in the 1980s the combination chemotherapy regimen of doxorubicin, bleomycin, vinblastine, and dacarbazine (ABVD) supplanted previous treatment strategies such as radiation therapy alone and nitrogen mustard, vincristine, procarbazine, and prednisone (MOPP) and has remained the standard of care for $\mathrm{HL}$ ever since [43-49]. However, despite the commonplace use of this regimen across most of the eras studied, HL survival has serially improved. We speculate that an increase in the access to care, improvement of diagnostic tools such as computed tomography and positron emission tomography scans, and efficacy of supportive care may be responsible for these increases in survival. A Spanish study found that patients treated before 1980 were 3.5 times more likely to die of infection and 7.1 times more likely to die of toxicity than those treated after 1980 [50]. Another reason for the improved survival in recent years could be due to improved diagnostic accuracy and elimination of the misdiagnosis of HL cases as NHL in the earlier years. The greatest additional improvements in HL survival are likely to come from identifying poor risk subgroups and developing management approaches specific for these populations that do not benefit from standard therapy. Studies such as this one help to identify subgroups (black patients, patients 45 years or older, and patients with stage IV disease) where specific interventions such as improving access to care, adding biological therapies, and intensifying initial therapy may be explored to improve survival for these poor-risk populations.

\section{Acknowledgment}

This work is funded by grants from the American Society of Hematology and the Leukemia \& Lymphoma Society.

\section{References}

[1] B. Schnitzer, "Hodgkin lymphoma," Hematology/Oncology Clinics of North America, vol. 23, no. 4, pp. 747-768, 2009.

[2] J. Burke, "Hodgkin's disease: histopathology and differential diagnosis," in Neoplastic Hematopathology, D. Knowles, Ed., pp. 497-533, Williams and Wilkins, Baltimore, Md, USA, 1992.

[3] American Cancer Society, "Cancer Facts \& Figures 2010," http: //www.cancer.org/acs/groups/content/@nho/documents/document/acspc-024113.pdf.

[4] V. T. Devita Jr., A. A. Serpick, and P. P. Carbone, "Combination chemotherapy in the treatment of advanced Hodgkin's disease," Annals of Internal Medicine, vol. 73, no. 6, pp. 881$895,1970$.

[5] H. Brenner, A. Gondos, and D. Pulte, "Ongoing improvement in long-term survival of patients with Hodgkin disease at all ages and recent catch-up of older patients," Blood, vol. 111, no. 6, pp. 2977-2983, 2008.

[6] S. L. Glaser and J. L. Hsu, "Hodgkin's disease in Asians: incidence patterns and risk factors in population-based data," Leukemia Research, vol. 26, no. 3, pp. 261-269, 2002.

[7] A. Maggioncalda, N. Malik, P. Shenoy, M. Smith, R. Sinha, and C. R. Flowers, "Clinical, molecular, and environmental risk factors for hodgkin lymphoma," Advances in Hematology, vol. 2011, Article ID 736261, 10 pages, 2011.

[8] L. A. G. Ries, C. L. Kosary, B. F. Hankey, B. A. Miller, A. Harras, and B. K. Edwards, Eds., SEER Cancer Statistics Review, 19731994, National Cancer Institute, Bethesda, Md, USA, 1997.

[9] D. Pulte, A. Gondos, and H. Brenner, "Trends in 5- and 10 -year survival after diagnosis with childhood hematologic malignancies in the United States, 1990-2004," Journal of the National Cancer Institute, vol. 100, no. 18, pp. 1301-1309, 2008.

[10] Surveillance, Epidemiology, and End Results (SEER) Program (http://www.seer.cancer.gov/): SEER*Stat Database: Incidence-SEER 13 Regs Limited-Use, Nov 2009 Sub (19922007) < Katrina/Rita Population Adjustment>-Linked To County Attributes-Total U.S., 1969-2007 Counties, National Cancer Institute, DCCPS, Surveillance Research Program, Cancer Statistics Branch, released April 2010, based on the November 2009 submission.

[11] B. F. Hankey, L. A. Ries, and B. K. Edwards, "The surveillance, epidemiology, and end results program: a national resource," Cancer Epidemiology Biomarkers and Prevention, vol. 8, no. 12, pp. 1117-1121, 1999.

[12] Surveillance, Epidemiology, and End Results (SEER) Program (http://www.seer.cancer.gov/): SEER*Stat Database: Incidence-SEER 17 Regs Limited-Use + Hurricane Katrina impacted Louisiana Cases, Nov 2009 Sub (2000-2007) $<$ Katrina/Rita Population Adjustment $>$ - Linked To County Attributes-Total U.S., 1969-2007 Counties, National Cancer Institute, DCCPS, Surveillance Research Program, Cancer Statistics Branch, released April 2010, based on the November 2009 submission.

[13] C. L. Percy, J. W. Berg, and L. B. Thomas, Eds., Manual of Tumor Nomenclature and Coding, American Cancer Society, New York, NY, USA, 1968.

[14] ICD-O International Classification of Diseases for Oncology, World Health Organization, Geneva, Switzerland, 1976.

[15] C. L. Percy, J. W. Berg, and L. B. Thomas, Eds., International Classification of Diseases for Oncology, World Health Organization, Geneva, Switzerland, 1990. 
[16] N. L. Harris, E. S. Jaffe, H. Stein et al., "A revised EuropeanAmerican classification of lymphoid neoplasms: a proposal from the International Lymphoma Study Group," Blood, vol. 84, no. 5, pp. 1361-1392, 1994.

[17] E. S. Jaffe, N. L. Harris, H. Stein, and J. W. Vardiman, Eds., World Health Organization Classification of Tumours. Pathology and Genetics of Tumours of Haematopoietic and Lymphoid Tissues, IARC Press, Lyon, France, 2001.

[18] S. H. Swerdlow, E. Campo, N. L. Harris et al., Eds., WHO Classification of Tumours of Haematopoietic and Lymphoid Tissues, IARC Press, Lyon, France, 4th edition, 2008.

[19] C. Percy, A. Fritz, and L. Ries, Conversion of Neoplasms by Topography and Morphology from the International Classification of Diseases for Oncology, Second Edition to International Classification of Diseases for Oncology, 3rd edition, 2001.

[20] L. M. Morton, S. S. Wang, S. S. Devesa, P. Hartge, D. D. Weisenburger, and M. S. Linet, "Lymphoma incidence patterns by WHO subtype in the United States, 1992-2001," Blood, vol. 107, no. 1, pp. 265-276, 2006.

[21] A. Fritz, C. Percy, A. Jack et al., Eds., International Classification of Diseases for Oncology, World Health Organization, Geneva, Switzerland, 3rd edition, 2000.

[22] L. M. Morton, J. J. Turner, J. R. Cerhan et al., "Proposed classification of lymphoid neoplasms for epidemiologic research from the Pathology Working Group of the International Lymphoma Epidemiology Consortium (InterLymph)," Blood, vol. 110, no. 2, pp. 695-708, 2007.

[23] NAACCR Race and Ethnicity Work Group, NAACCR Asian Pacific Islander Identification Algorithm [NAPIIA v1.2.1], North American Association of Central Cancer Registries, Springfield, Ill, USA, 2010.

[24] L. A. G. Ries, M. P. Eisner, C. L. Kosary et al., Eds., SEER Cancer Statistics Review, 1975-2001, National Cancer Institute, Bethesda, Md, USA, 2004, http://seer.cancer.gov/csr/ 1975_2001/.

[25] J. C. Day, "Population Projections of the United States by Age, Sex, Race, and Hispanic Origin: 1995 to 2050," U.S. Bureau of the Census, Current Population Reports, P25-1130, U.S. Government Printing Office, Washington, DC, USA, 1996, http://www.census.gov/prod/1/pop/p25-1130/p251130.pdf.

[26] C. C. Brown, "The statistical comparison of relative survival rates," Biometrics, vol. 39, no. 4, pp. 941-948, 1983.

[27] C. A. Clarke, D. M. Undurraga, P. J. Harasty, S. L. Glaser, L. M. Morton, and E. A. Holly, "Changes in cancer registry coding for lymphoma subtypes: reliability over time and relevance for surveillance and study," Cancer Epidemiology Biomarkers and Prevention, vol. 15, no. 4, pp. 630-638, 2006.

[28] C. A. Clarke, S. L. Glaser, and A. W. Prehn, "Age-specific survival after Hodgkin's disease in a population-based cohort (United States)," Cancer Causes and Control, vol. 12, no. 9, pp. 803-812, 2001.

[29] L. J. Medeiros and T. C. Greiner, “Hodgkin's disease," Cancer, vol. 75, no. 1, pp. 357-369, 1995.

[30] B. Macmahon, "Epidemiological evidence of the nature of Hodgkin's disease," Cancer, vol. 10, no. 5, pp. 1045-1054, 1957.

[31] H. Hjalgrim, K. Rostgaard, P. C.D. Johnson et al., "HLA-A alleles and infectious mononucleosis suggest a critical role for cytotoxic T-cell response in EBV-related Hodgkin lymphoma," Proceedings of the National Academy of Sciences of the United States of America, vol. 107, no. 14, pp. 6400-6405, 2010.

[32] S. L. Glaser, R. J. Lin, S. L. Stewart et al., "Epstein-Barr virusassociated Hodgkin's disease: epidemiologic characteristics in international data," International Journal of Cancer, vol. 70, no. 4, pp. 375-382, 1997.
[33] W. Cozen, A. S. Hamilton, P. Zhao et al., "A protective role for early oral exposures in the etiology of young adult Hodgkin lymphoma," Blood, vol. 114, no. 19, pp. 4014-4020, 2009.

[34] T. H. M. Keegan, S. L. Glaser, C. A. Clarke et al., "EpsteinBarr virus as a marker of survival after Hodgkin's lymphoma: a population-based study," Journal of Clinical Oncology, vol. 23, no. 30, pp. 7604-7613, 2005.

[35] P. J. Shenoy, N. Malik, A. Nooka et al., "Racial differences in the presentation and outcomes of diffuse large B-cell lymphoma in the United States," Cancer. In press.

[36] S. L. Glaser, C. A. Clarke, M. L. Gulley et al., "Populationbased patterns of human immunodeficiency virus-related Hodgkin lymphoma in the greater San Francisco bay area, 1988-1998," Cancer, vol. 98, no. 2, pp. 300-309, 2003.

[37] R. J. Biggar, E. S. Jaffe, J. J. Goedert, A. Chaturvedi, R. Pfeiffer, and E. A. Engels, "Hodgkin lymphoma and immunodeficiency in persons with HIV/AIDS," Blood, vol. 108, no. 12, pp. 37863791, 2006.

[38] C. U. Oramasionwu, C. M. Brown, L. Ryan, K. A. Lawson, J. M. Hunter, and C. R. Frei, "HIV/AIDS disparities: the mounting epidemic plaguing US blacks," Journal of the National Medical Association, vol. 101, no. 12, pp. 1196-1204, 2009.

[39] A. N. AbouYabis, P. J. Shenoy, M. J. Lechowicz, and C. R. Flowers, "Incidence and outcomes of the peripheral T-cell lymphoma subtypes in the United States," Leukemia and Lymphoma, vol. 49, no. 11, pp. 2099-2107, 2008.

[40] P. J. Shenoy, K. Bumpers, N. King et al., "Black/white differences in the treatment and outcomes of diffuse large b cell lymphoma: a matched cohort analysis," ASH Annual Meeting Abstracts, vol. 114, no. 22, p. 1392, 2009.

[41] M. Wang, K. D. Burau, S. Fang, H. Wang, and X. L. Du, "Ethnic variations in diagnosis, treatment, socioeconomic status, and survival in a large population-based cohort of elderly patients with non-Hodgkin lymphoma," Cancer, vol. 113, no. 11, pp. 3231-3241, 2008.

[42] D. Hasenclever and V. Diehl, "A prognostic score for advanced Hodgkin's disease. International Prognostic Factors Project on Advanced Hodgkin's Disease," The New England Journal of Medicine, vol. 339, no. 21, pp. 1506-1514, 1998.

[43] P. G. Gobbi, A. Levis, T. Chisesi et al., "ABVD versus modified Stanford V versus MOPPEBVCAD with optional and limited radiotherapy in intermediate- and advanced-stage Hodgkin's lymphoma: final results of a multicenter randomized trial by the Intergruppo Italiano Linfomi," Journal of Clinical Oncology, vol. 23, no. 36, pp. 9198-9207, 2005.

[44] A. Engert, J. Franklin, H. T. Eich et al., "Two cycles of doxorubicin, bleomycin, vinblastine, and dacarbazine plus extended-field radiotherapy is superior to radiotherapy alone in early favorable Hodgkin's lymphoma: final results of the GHSG HD7 trial," Journal of Clinical Oncology, vol. 25, no. 23, pp. 3495-3502, 2007.

[45] P. J. Hoskin, L. Lowry, A. Horwich et al., "Randomized comparison of the Stanford V regimen and ABVD in the treatment of advanced Hodgkin's lymphoma: United Kingdom National Cancer Research Institute Lymphoma Group Study ISRCTN 64141244," Journal of Clinical Oncology, vol. 27, no. 32, pp. 5390-5396, 2009.

[46] D. B. Duggan, G. R. Petroni, J. L. Johnson et al., "Randomized comparison of ABVD and MOPP/ABV hybrid for the treatment of advanced Hodgkin's disease: report of an Intergroup trial," Journal of Clinical Oncology, vol. 21, no. 4, pp. 607-614, 2003. 
[47] G. P. Canellos, L. Nadler, and T. Takvorian, "Autologous bone marrow transplantation in the treatment of malignant lymphoma and Hodgkin's disease," Seminars in Hematology, vol. 25, no. 2, pp. 58-65, 1988.

[48] G. Bonadonna and A. Santoro, "ABVD chemotherapy in the treatment of Hodgkin's disease," Cancer Treatment Reviews, vol. 9, no. 1, pp. 21-35, 1982.

[49] G. P. Canellos, J. R. Anderson, K. J. Propert et al., "Chemotherapy of advanced Hodgkin's disease with MOPP, ABVD, or MOPP alternating with ABVD," The New England Journal of Medicine, vol. 327, no. 21, pp. 1478-1484, 1992.

[50] M. Provencio, I. Millán, P. España et al., "Analysis of competing risks of causes of death and their variation over different time periods in hodgkin's disease," Clinical Cancer Research, vol. 14 , no. 16 , pp. 5300-5305, 2008. 


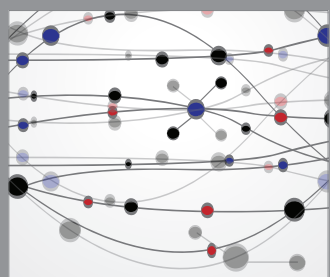

The Scientific World Journal
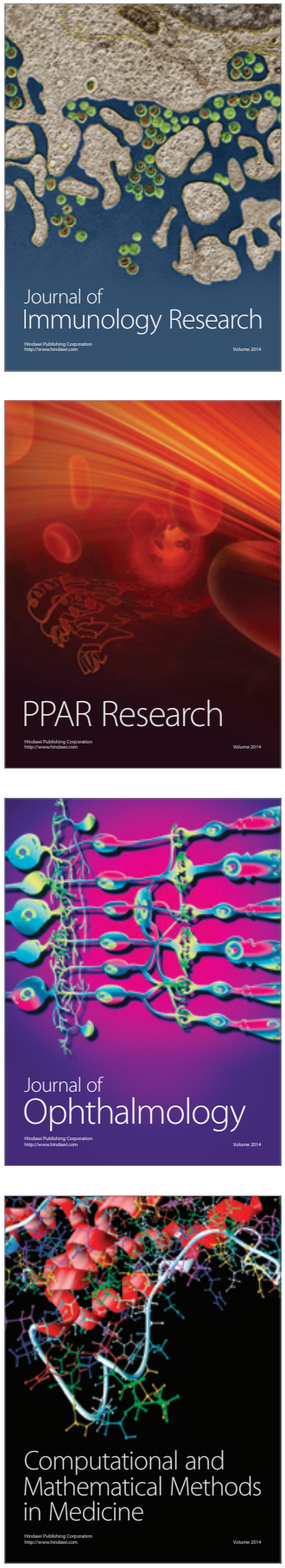

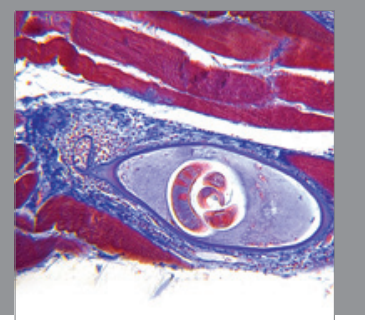

Gastroenterology

Research and Practice
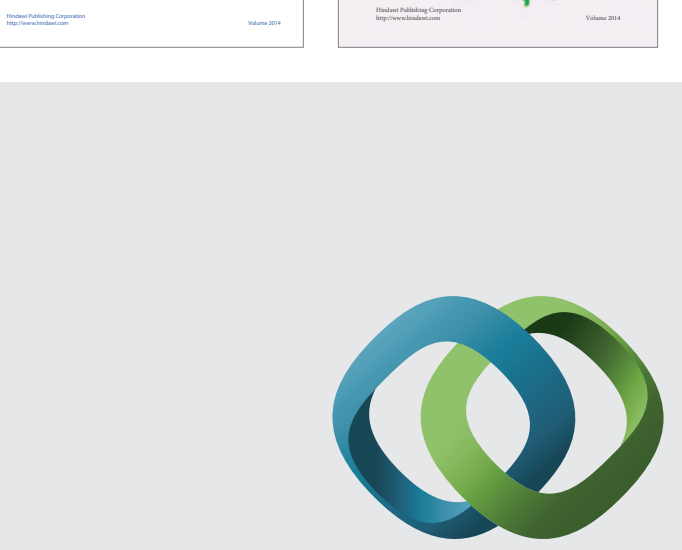

\section{Hindawi}

Submit your manuscripts at

http://www.hindawi.com
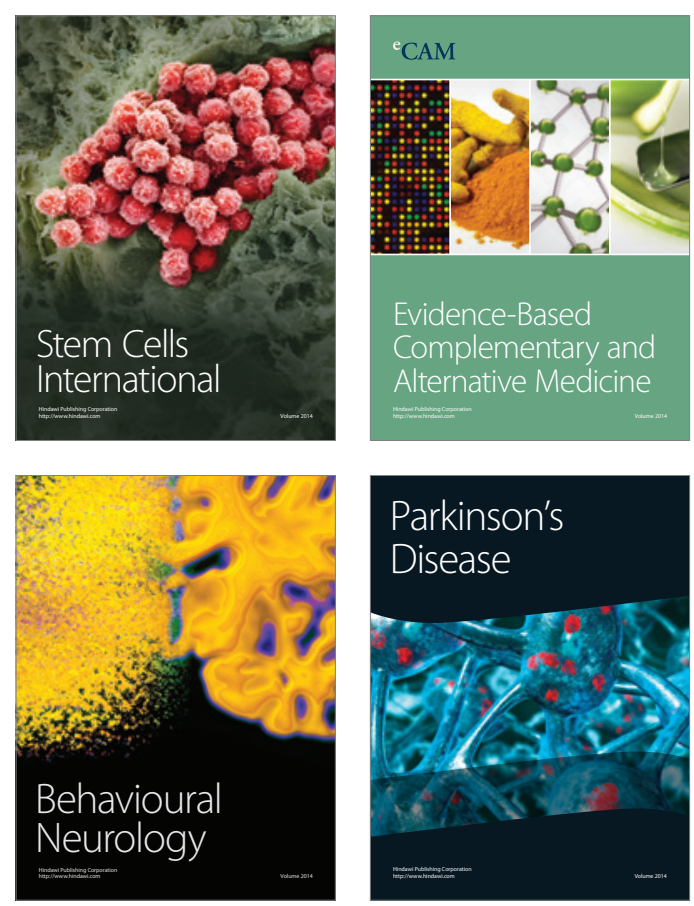

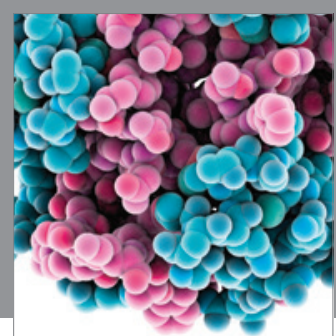

Journal of
Diabetes Research

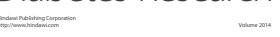

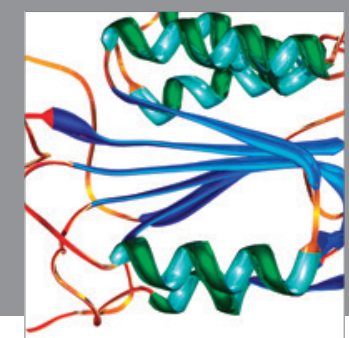

Disease Markers
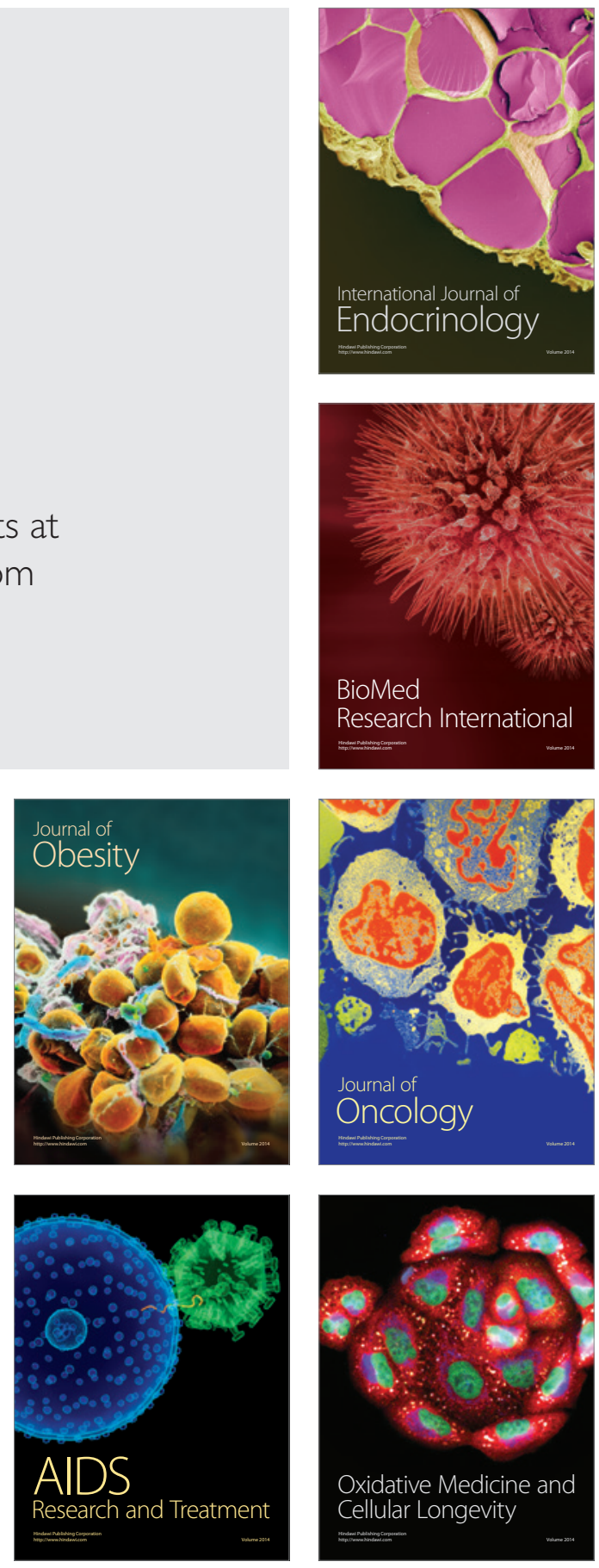